\title{
New arguments against the charge transfer mechanism in the hydrogen transfer reaction between nitromethane and hydrogen sulphide
}

\author{
Dmitriy Fomichev*, Dmitriy Ovsyannikov and Sergei Zelentsov \\ Lobachevsky State University of Nizhni Novgorod \\ * xomachiner@gmail.com
}

Received: 25.10.17; Published: 1.11.17

\begin{abstract}
In our recent work we have made a conclusion that in the radical reaction between nitromethane and hydrogen sulphide the formation of the anion-radical does not happen. This conclusion was not expected due to oposite results that can be found in many experimental papers describing the similiar reactions with nitronaphtalene and amines. So, the additional research has been made using RT-TDDFT simulation at the PBE0/6-311++G** theory level. The simulation has shown no charge oscillations between reactants, that can be interpreted as an argument against the charge transfer mechanism. The study of correlation between solvent polarity and energetic parameters of reaction has been made using the SMD and COSMO above the DFT PBE0/6-311++ $G^{* *}$ calculation has shown non-polar nature of the reaction, that also might be used as an argument against the anion-radical formation mechanism.
\end{abstract}

Keywords: charge transfer; nitrocompounds; triplet state; sulphide; RT-TDDFT

\section{Introduction}

The developing of suitable photooxidizing agent could be demanded in many industries. Nitrocompounds are a good candidate due to their fast ISC to the triplet manifold[1] and the demand of their recycling.

Previous experimental research has shown[2] that the aromatic nitrocompounds tend to receive an electron from a reductant before the actual protonation in the photoreduction mechanism. Another research[3] describes the formation mechanism of of the nitroaromatic anion-radical in pair with 1,4-diazabicyclo[2.2.2]octane cation. The charge transfer has been also detected in sulphide oxidation reaction[4] in aqueous solution.

Despite these results our recent ab-initio quantum chemical study[5] has not revealed any charge transfer preceeding of the hydrogen transfer in the hydrogen sulphide oxidation reaction. Such an inconvergency of results can be explained by a numerous factors including the solvent influence in experimental data, incorrect description of the triplet state reactions by the model used, etc. So, in the present work we have tried to eluminate some of those factors by performing RT-TDDFT simulation of the electron density in reagents and by studying solvent influence on the reaction energetic parameters using SMD and COSMO approach.

\section{Results}

The RT-TDDFT simulation was shown absolutely no charge oscillations between reactants. But there was observed dipole moment oscillations. So the UV/Vis-spectra was calculated and plotted for these oscillations and it can be found at fig. 1. It could be seen from the fig. 1 that the spectra contains the certain peak between $380 \mathrm{~nm}$ and $390 \mathrm{~nm}$ and probably two more around $500 \mathrm{~nm}$ and $640 \mathrm{~nm}$. 


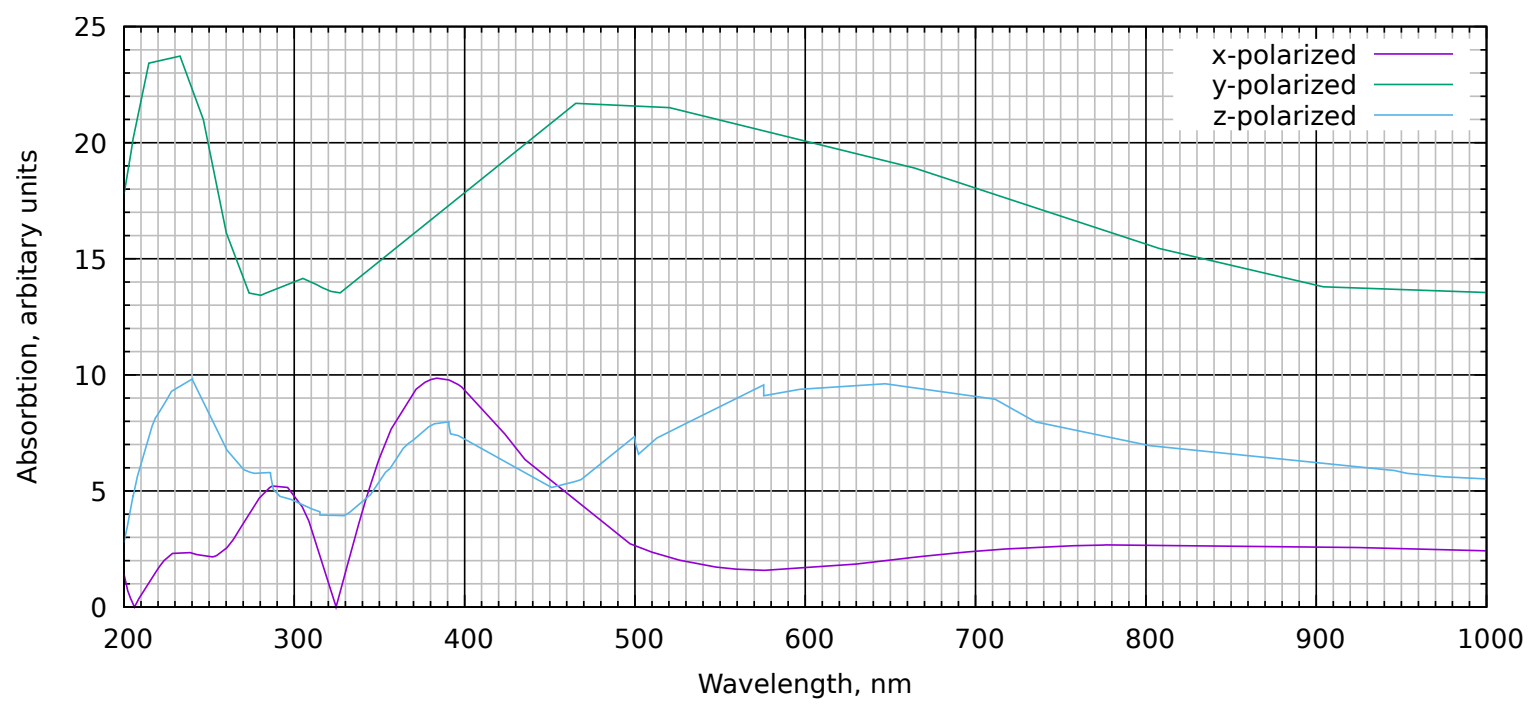

Figure 1. The UV/Vis-spectra obtained from the dipole moment oscillation in reactants system and ploted for $\mathrm{x}, \mathrm{y}$ and $\mathrm{z}$ polarizations

The solvent influence on the reaction has been shown at fig. 2. As it can be seen from the graph, there is no dependency can be found between solvents dielectic constant and activation energy or enthalpy values. The activation energy of the reaction in all solvents considered is about $4.5 \mathrm{kcal} \mathrm{mol}^{-1}$ and the difference between the energies does not exceed $1 \mathrm{kcal} \mathrm{mol}^{-1}$ for most of solvents. The enthalpy of the reaction also shows very little variety of values and is around $-15.5 \mathrm{kcal} \mathrm{mol}^{-1}$. The only exception from that tendency is water solvent. The activation energy of the reaction in water is much lower than in other solvents. On the contrary, the entalpy of the reaction in water solvent is higher on more than $3 \mathrm{kcal} \mathrm{mol}^{-1}$ than other solvents.

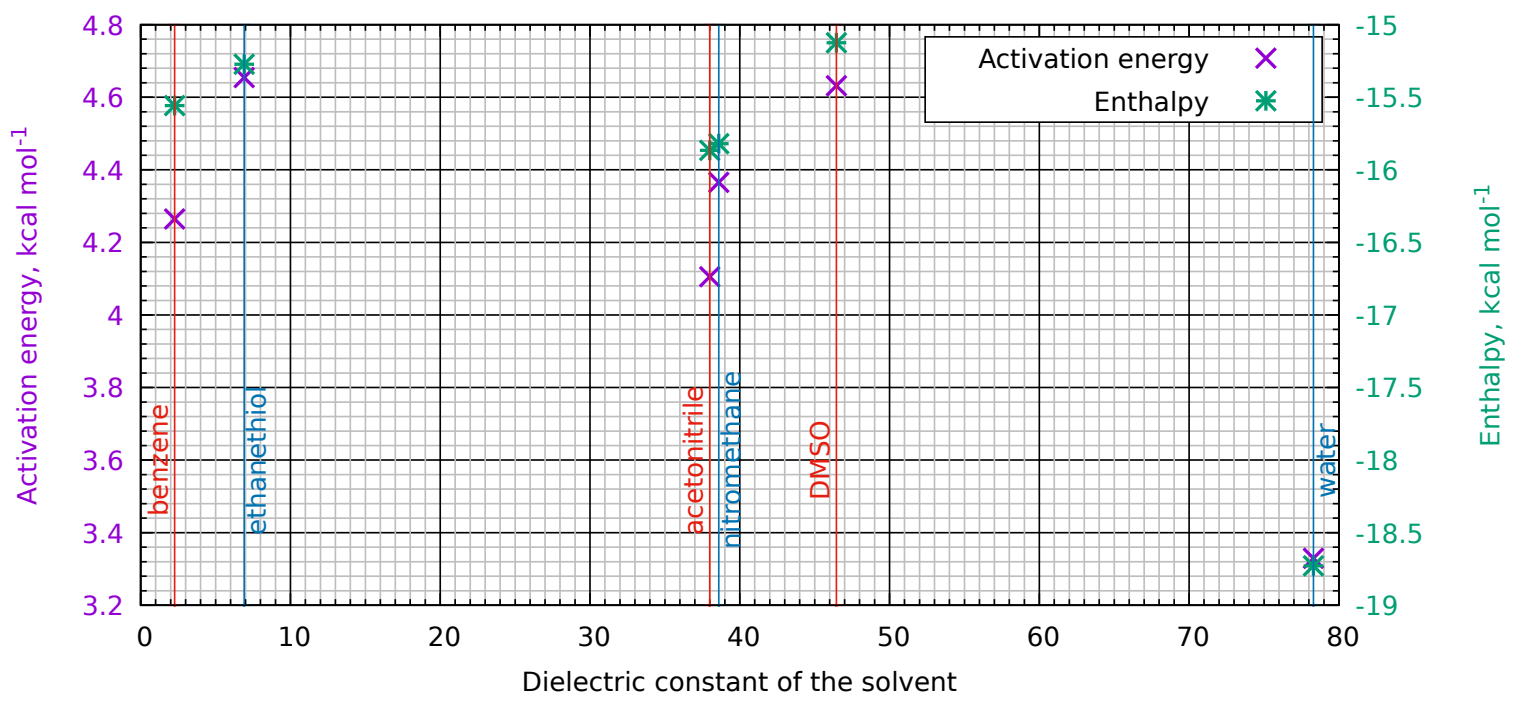

Figure 2. The activation energy and enthalpy dependence from solvents dielectric constant.

It is important to notice that the activation energies in all considered solvents are higher than activation energy of the same reaction in the gas phase which value is equal to $3.6 \mathrm{kcal} \mathrm{mol}^{-1}$ according to our previous studies.[5] 


\section{Discussion}

Despite of the fact that the charge transfer was not detected by the RT-TDDFT calculation, the dipole moments oscillations might be treated as an attribute of the charge transfer. It is wondering fact that the peaks at the UV/Vis-spectra shown on the fig. 1 is similiar with the peaks observed in [3]. The peak at $390 \mathrm{~nm}$ might be associated with the triplet nitro group absorbtion, as soon as it is present with little variations in both of nitronaphtalene spectras from paper mentioned above and nitromethane spectra obtained in this study. So we assume that dipole moment oscillations observed in RT-TDDFT calculation results can not be considered as an argument for the charge transfer, especcialy taking into account the abscence of the actual charge oscillations between reactants in the calculation results.

Another argument against the charge transfer is lies in independence of energetic parameters of the reaction from the dielectric constant of the solvent in range from 0 to 46 . The decreasing of the activation energy and increasing of the enthalpy of the reaction were an unexpected result and requires futher investigation to be performed as well as influence of solvents with dielectric constants lying in range from 46 to 80 . Anyway if the anion-radical were formed during the reaction the changing of the dielectric constant of the solvent should have a significant impact on energetic parameters of the reaction. But in our model we have not observed any noticable energy changes in wide range of solvents.

\section{Materials and Methods}

All calculations described in this paper were performed in the North-West Chemistry software package.[6] The study of the solvent influence on energetic parameters of the reaction is done via SMD and COSMO approaches above the DFT PBE0/6-311++G** theory level. Such a method was selected due to its acceptable description of the reagents as it was pointed out in [7]. Activation energies were calculated taking into account with zero-point energy correction. For enthalpies calculation the enthalpy correction to energy was also used.

The RT-TDDFT calculation[8] was performed in the following way. First, reactants were optimized to minimum in both of separated from each other and united to one system using TDDFT on PBE0/6-311++ $G^{* *}$ theory level. Then the RT-TDDFT simulation of united reactants was started for $15 \mathrm{fs}$ using molecular orbitals obtained from optimization of separated reactants. After completion of the simulation the dependence of charge on each molecular fragment from time was used to made a conclusion about the charge transfer. The UV/Vis-spectra was obtained by performing Fourier transformation of dipole moment values in the way described in NWChem manual. ${ }^{1}$

Acknowledgments: Authors are grateful to the Volga Research and Education Center for Supercomputer Technologies for providing access to resources of the Lobachevsky supercomputer.

\section{Abbreviations}

The following abbreviations are used in this manuscript:

COSMO COnductor-like Screening MOdel

ISC InterSystem Crossing

PES Potential Energy Surface

RT-TDDFT Real-Time Time-Dependent Density Functional Theory

SMD Solvation Model based on Density

1 http://www.nwchem-sw.org/index.php/Release66:RT-TDDFT\#Absorption_spectrum_of_water 


\section{References}

1. Zugazagoitia, J.S.; Almora-Díaz, C.X.; Peon, J. Ultrafast Intersystem Crossing in 1-Nitronaphthalene. An Experimental and Computational Study. The Journal of Physical Chemistry A 2008, 112, 358-365. bibtex: zugazagoitia_ultrafast_2008.

2. Cu, A.; Testa, A.C. Evidence for electron transfer in the photoreduction of aromatic nitro compounds. Journal of the American Chemical Society 1974, 96, 1963-1965. bibtex: cu_evidence_1974-1.

3. Görner, H.; Döpp, D. Photoreduction induced by electron transfer from di- and trialkylamines to the triplet state of nitronaphthalenes in polar media. Journal of the Chemical Society, Perkin Transactions 2 2002, pp. 120-125. bibtex: gorner_photoreduction_2002.

4. Hashimoto, S.; Sunamoto, J.; Sato, K. The Alkaline Sulfide Reduction of Aromatic Nitro Compounds. XI. The Autocatalytic Reaction of the Monosulfide to the Disulfide in Aqueous Solution. Bulletin of the Chemical Society of Japan 1967, 40, 2860-2866.

5. Fomichev, D.; Gubochkin, N.; Ovsyannikov, D.; Zelentsov, S. The reaction of the hydrogen sulphide with the triplet nitromethane in the gas phase: the mechanism and products evaluation. MDPI, 2016, p. e009.

6. Valiev, M.; Bylaska, E.J.; Govind, N.; Kowalski, K.; Straatsma, T.P.; Van Dam, H.J.J.; Wang, D.; Nieplocha, J.; Apra, E.; Windus, T.L.; de Jong, W.A. NWChem: A comprehensive and scalable open-source solution for large scale molecular simulations. Computer Physics Communications 2010, 181, 1477-1489. bibtex: valiev_nwchem:_2010.

7. Fomichev, D.A.; Zelentsov, S.V. Theoretical study of photochemical oxidation of organic sulfides and hydrogen sulfide in the presence of nitro compounds. High Energy Chemistry 2015, 49, 104-110. bibtex: fomichev_theoretical_2015.

8. Lopata, K.; Govind, N. Modeling Fast Electron Dynamics with Real-Time Time-Dependent Density Functional Theory: Application to Small Molecules and Chromophores. Journal of Chemical Theory and Computation 2011, 7, 1344-1355.

(C) 2017 by the authors. Licensee MDPI, Basel, Switzerland. This article is an open access article distributed under the terms and conditions of the Creative Commons Attribution (CC BY) license (http:/ / creativecommons.org/licenses/by/4.0/). 